\title{
Viral respiratory tract infections in young children with cystic fibrosis: a prospective full-year seasonal study
}

\author{
Mathilde Eymery ${ }^{1,8^{*}}$ (D), Florence Morfin ${ }^{2,3,4}$, Anne Doleans-Jordheim, ${ }^{5,6}$, Marie Perceval ${ }^{1}$, Camille OhImann ${ }^{1}$,
} Catherine Mainguy ${ }^{1}$ and Philippe Reix ${ }^{1,7}$

\begin{abstract}
Background: Viral respiratory tract infections are common during early childhood. How they impact cystic fibrosis lung disease history in young children is poorly known. The principal aim of our study was to determinate respiratory tract infections frequency in this cystic fibrosis young population. Secondary outcomes were nature of viral agents recovered and impact of such infections.

Methods: We conducted a prospective cohort study of 25 children affected by cystic fibrosis and aged less than 2 years. Nasal samplings were taken systematically monthly or bimonthly with additional samples taken during respiratory tract infections episodes. Ten pathogens were tested by a combination of five duplex RTPCRs or PCRs: influenza $A$ and $B$, respiratory syncytial virus (RSV), metapneumovirus (MPV), rhinovirus/enterovirus (RV/EV)), coronavirus (HKU1, NL63, 229E and OC43), parainfluenza virus (1-4), adenovirus and bocavirus (Respiratory MultiWell System MWS r-gene ${ }^{\oplus}$, BioMérieux, Marcy l'Étoile, France). Cycle thresholds (CTs) were reported for all positive samples and considered positive for values below 40. Quantitative variables were compared using a nonparametric statistical test (Wilcoxon signed rank for paired comparisons). Pearson's correlation coefficient ( $r$ ) was used to assess relationships between two variables. Statistical analyses were performed using SAS v9.4 (SAS Institute, Cary, NC, USA) or GraphPad Prism V6.00 (GraphPad Software, La Jolla, CA, USA). The significance level was set at 0.05.

Results: The mean age at inclusion was $9.6 \pm 6.7$ months. The patients had $3.4 \pm 1.7$ respiratory tract infections episodes per child per year. Forty-four respiratory tract infections (69\%) were associated with virus: rhinovirus and enterovirus (RV/EV) were implied in $61 \%$ of them and respiratory syncytial virus (RSV) in 14\%. Only one patient required hospitalization for lower respiratory tract infections. $86 \%$ of the patients were treated by antibiotics for a mean of $13.8 \pm 6.2$ days. RSV infections $(n=6)$ were usually of mild severity.

Conclusions: Respiratory tract infections in young children with cystic fibrosis were of mild severity, rarely requiring hospitalization. Unsurprisingly, RV/EV were the most frequent agents. RSV-related morbidity seems low in this population. This raises the question of the usefulness of RSV preventive medication in this young population.
\end{abstract}

Keywords: Children, Respiratory virus, Cystic fibrosis

\footnotetext{
* Correspondence: mathilde.eymery@chu-lyon.fr; mathey18@hotmail.com

${ }^{1}$ Service de pneumologie pédiatrique et CRCM enfant, Hôpital Femme Mère

Enfant, Hospices civils de Lyon, Bron, France

${ }^{8}$ Centre de ressources et de compétence pour la Mucoviscidose, 59

boulevard Pinel, 69677 BRON CEDEX, France

Full list of author information is available at the end of the article
}

(C) The Author(s). 2019 Open Access This article is distributed under the terms of the Creative Commons Attribution 4.0 International License (http://creativecommons.org/licenses/by/4.0/), which permits unrestricted use, distribution, and reproduction in any medium, provided you give appropriate credit to the original author(s) and the source, provide a link to the Creative Commons license, and indicate if changes were made. The Creative Commons Public Domain Dedication waiver (http://creativecommons.org/publicdomain/zero/1.0/) applies to the data made available in this article, unless otherwise stated. 


\section{Background}

Cystic fibrosis (CF) is a rare genetic condition affecting more than 90,000 people worldwide. Despite implementation of neonatal newborn screening programs in some countries and improvement in patient care over the last few years, CF-related lung disease remains the main cause of morbidity-mortality in these patients. The role of bacterial pathogens such as Staphylococcus aureus (SA) and Pseudomonas aeruginosa (PA) has been extensively studied for several decades whereas the respiratory viruses pathogenesis remained little explored. The availability of new diagnostic molecular tests to detect virus infections has recently boosted the interest in evaluating their impact during pathological conditions such as CF in children [1-9] and in adults [10-15].

There is however a critical lack of data describing viral respiratory tract infections (RTIs) in young children with CF aged less than 2 years, when RTIs are particularly frequent and potentially more severe at this early period of life. Some studies conducted in the late 1980s and 1990s have drawn a pessimistic scenario of viral RTIs in this vulnerable population that is no longer observed. In these seminal studies, clinical manifestations were described as severe, frequently requiring hospitalization and prolonged oxygen supply or mechanical ventilation [16-18]. Some patients were described as acquiring pathogens such as PA in the weeks or months following RTI [18, 19]. At that time, respiratory syncytial virus (RSV) was found to be the most frequently recovered virus, but diagnostic tools were limited to immunological assays and viral culture, thus limiting the spectrum of virus that may be recovered. Recent studies have shown that RSV epidemics may be associated with the occurrence of more pulmonary exacerbations [20], but RSV is rarely responsible for hospitalization because of respiratory events in CF patients [21, 22].

We designed a prospective cohort study to better explore the frequency of RTI, along with their severity, the nature of viral agents recovered and the associated short-term outcomes.

\section{Material and methods}

\section{Patient recruitment}

Between March 2015 and April 2016, 33 families were contacted. Eight refused to participate (three because repeated nasal swab samplings were required, two because of the overall burden of the study, two for unexplained reasons, one for a language barrier issue). Finally, 25 young children with CF aged less than 24 months were recruited to participate in the study. All had confirmed CF based on compatible clinical features associated with two positive sweat tests (sweat chloride concentration $\geq 60 \mathrm{mmol} / \mathrm{L}$ ) and/or two CFTR gene mutations. None received palivizumab because it is not used in our current practice.
Study design, ethics, objectives and outcome measures This was a prospective cohort study conducted in a single pediatric center (Lyon, France). The study was approved by the local Institutional Review Board (Comité Consultatif de Protection des Personnes dans la Recherche Biomédicale, Lyon; No. 2014-AO1387640). Parents or legal guardians gave their assent and signed an informed consent form before their children entered the study.

The primary outcome measure was the frequency of RTIs per year. The secondary outcomes measured during the year of follow-up were: (1) virus identification by multiplex PCR; (2) number of hospitalizations, number of oral/intravenous antibiotic courses, steroids, bronchodilator use, number of days of parental work absenteeism, unscheduled outpatient visits; (3) difference in weight Z-scores between the start and the end of the study; (4) comparison between virus-negative and -positive RTIs for age and weight Z-score at inclusion, difference in weight Z-scores between the beginning and end of the study period, total number of RTIs, cumulative number of days of antibiotics; (5) percentage of samples positive for PA and SA out of the the total number of samples taken routinely during the study, new isolation of a pathogen.

\section{RTI identification, nasal swab sampling and viral analysis}

It is routine practice in France for chest physiotherapists to visit patients at home to ensure regular chest physiotherapy and respiratory surveillance. As already reported [23] we thought that proper nasal sampling and filling out diaries by parents may be difficult to perform repeatedly over a long period of time; chest physiotherapists were therefore involved in the study to take nasal samples and recognize RTIs. Parents and chest physiotherapists were instructed to recognize RTI using a modified clinical score already used in a previous study conducted by van Ewijk and al in young children with CF [9]. There, the score was systematically performed twice weekly by parents, while in our study it was used on request by parents and physiotherapists to detect or confirm a possible RTI episodes revealed by the occurrence of symptoms. Briefly, Upper and Lower respiratory tract infection (URTI and LRTI) symptoms as well as general signs such as fever were recorded and a score calculated. In case of doubt, parents could ask the research nurse (by phone) or their chest physiotherapist (during the home visit) to confirm their findings. When the score was over 2, a nasal sample was collected at home by the chest physiotherapist within a maximum 3 days after the beginning of the symptoms, or at the center when the episode was coinciding with a visit.

Furthermore, nasal swabs were systematically scheduled (even in absence of RTI signs) at the following frequencies depending on viral seasonality: every month 
from September 1st to March 31st and every 2 months from April 1st to October 31st during the year following inclusion in the study.

Nasopharyngeal samples were taken according to a recently described procedure using swabs (Sigma-Virocult ${ }^{\circ}$ M40 compliant. MWE. Corsham Wiltshire, UK) after saline instillation [24].

Respiratory specimen sampling (pharyngeal swabs) for bacterial cultures was performed at each center visit (four to six samples per patient per year depending on the age at inclusion). Bacterial cultures were performed on specific medias in order to identify major CF pathogens such as SA or PA. Colonies morphology, gram staining were than used for a first identification of each bacterial species. Than a MALDI-TOF-MS approach was done for the final identification.

Ten pathogens were tested by a combination of five duplex RT-PCRs or PCRs: influenza $A$ and $B$, respiratory syncytial virus (RSV), metapneumovirus (MPV), rhinovirus/enterovirus (RV/EV)), coronavirus (HKU1, NL63, 229E and OC43), parainfluenza virus (1-4), adenovirus and bocavirus (Respiratory Multi-Well System MWS r-gene ${ }^{\bullet}$, BioMérieux, Marcy l'Étoile, France). Cycle thresholds (CTs) were reported for all positive samples and considered positive for values below 40 .

\section{Statistical analysis}

Quantitative variables are reported as mean \pm standard deviation. Quantitative variables were compared using a nonparametric statistical test (Wilcoxon signed rank for paired comparisons). Pearson's correlation coefficient (r) was used to assess relationships between two variables. Statistical analyses were performed using SAS v9.4 (SAS Institute, Cary, NC, USA) or GraphPad Prism V6.00 (GraphPad Software, La Jolla, CA, USA). The significance level was set at 0.05 .

\section{Results}

\section{Patient characteristics at inclusion}

Twenty-five young children with a mean age of $9.6 \pm 6.7$ months (range, 2-22.9 months) were included (Table 1). Half of them had siblings in their immediate family environment. Half attended exclusively family daycare. None received palivizumab and 22 patients $(88 \%)$ were vaccinated against influenza virus. Sixty percent had commensal flora at inclusion.

\section{Primary outcome: RTI frequency}

Eighty-five RTIs were identified for an average of $3.4 \pm$ 1.7 RTIs per child per year. Virally proven RTIs were identified at an average of $1.8 \pm 1.5$ per child per year. There was no correlation between age at inclusion and the total number of RTIs (Pearson correlation, $r=-0.24$, $p=0.2353$ ) nor virus-positive RTIs (Pearson correlation,
Table 1 Patient characteristics at inclusion

\begin{tabular}{ll}
\hline Number of patients & 25 \\
\hline Gender (male/female) & $15 / 10$ \\
Premature birth $n$ ( $\%)$ & $5(20 \%)$ \\
Season at birth & \\
Spring & \\
Summer & $6(24 \%)$ \\
Fall & $6(24 \%)$ \\
$\quad$ Winter & $5(20 \%)$ \\
Maternal smoking during pregnancy & $8(32 \%)$ \\
Maternal atopy & $4(16 \%)$ \\
Parental smoking & $6(24 \%)$ \\
Siblings & $15(60 \%)$ \\
0 & \\
1 & $12(48 \%)$ \\
$\geq 2$ & $8(32 \%)$ \\
Daycare attendance & $5(20 \%)$ \\
& Family only: $13(52 \%)$ \\
& Small group: $8(32 \%)$ \\
& Collective: $4(16 \%)$
\end{tabular}

\begin{tabular}{|c|c|}
\hline \multicolumn{2}{|l|}{ Demographics at inclusion } \\
\hline \multicolumn{2}{|l|}{ CFTR genotype with } \\
\hline No CFTR function & $21(84 \%)$ \\
\hline Residual CFTR function & $5(16 \%)$ \\
\hline Unknown consequences & $0(0 \%)$ \\
\hline Age at inclusion (months) & $9.4 \pm 6.7$ \\
\hline Range & $2-22.9$ \\
\hline \multicolumn{2}{|l|}{ Anthropometrics (Z-scores) } \\
\hline Weight & $-1.3 \pm 1.4$ \\
\hline Height & $-1.1 \pm 1.5$ \\
\hline \multicolumn{2}{|l|}{ Vaccination coverage rates } \\
\hline DTPa-IPV/Hib ${ }^{d}$ & $25(100 \%)$ \\
\hline Influenza & $22(88 \%)$ \\
\hline RSV prophylaxis (palivizumab) & $0(0 \%)$ \\
\hline \multicolumn{2}{|l|}{ Bacteria recovered at inclusion } \\
\hline Oropharyngeal flora & $15(60 \%)$ \\
\hline Staphylococcus aureus & $8(32 \%)$ \\
\hline Pseudomonas aeruginosa & $1(4 \%)$ \\
\hline Haemophilus influenzae & $1(4 \%)$ \\
\hline \multicolumn{2}{|l|}{ Virus recovered at inclusion } \\
\hline None & $10(40 \%)$ \\
\hline RV/EV & $7(28 \%)$ \\
\hline Bocavirus & $4(16 \%)$ \\
\hline Influenzae & $2(8 \%)$ \\
\hline Metapneumovirus & $1(4 \%)$ \\
\hline Cytomegalovirus $^{\mathrm{e}}$ & $1(4 \%)$ \\
\hline RSV & $0(0 \%)$ \\
\hline \multicolumn{2}{|c|}{ 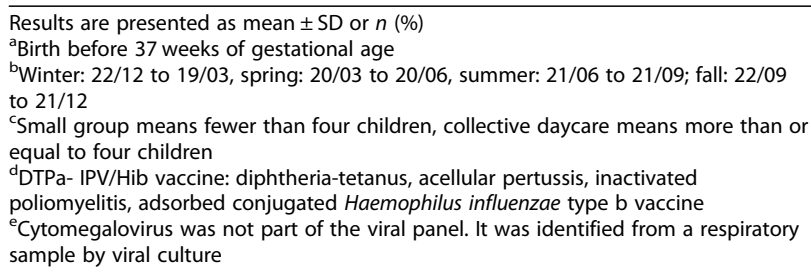 } \\
\hline
\end{tabular}


$r=0.065, p=0.6710$ ) (Fig. 1 correlation between age at inclusion and a. total respiratory tract infections or b. virus positive respiratory tract infection episodes). There were no specific clinical features related to viral-positive RTIs.

\section{Secondary outcomes}

\section{Viruses recovered during $R T I$}

During the year of follow-up, 242 samples were collected (153 at home and 89 during a visit to the center), a mean of $9.7 \pm 2.1$ samples per patient. Out of the 85 RTIs clinically identified, 64 samples (75\%) were collected during symptoms occurrence, while 21 samples were not realized. Out of 64 RTIs sampled, 44 (69\%) were associated with the identification of a viral agent (Fig. 2 flow chart of virus sample results).

The viruses recovered during viral RTIs were $R V / E V$ (27/44; 61\%), RSV (6/44; 14\%), adenovirus (6/44; 14\%), influenza (4/44; 9\%), bocavirus (3/44; 7\%), parainfluenzae (2/44; 5\%) and metapneumovirus (1/44; 2\%). Eight viral
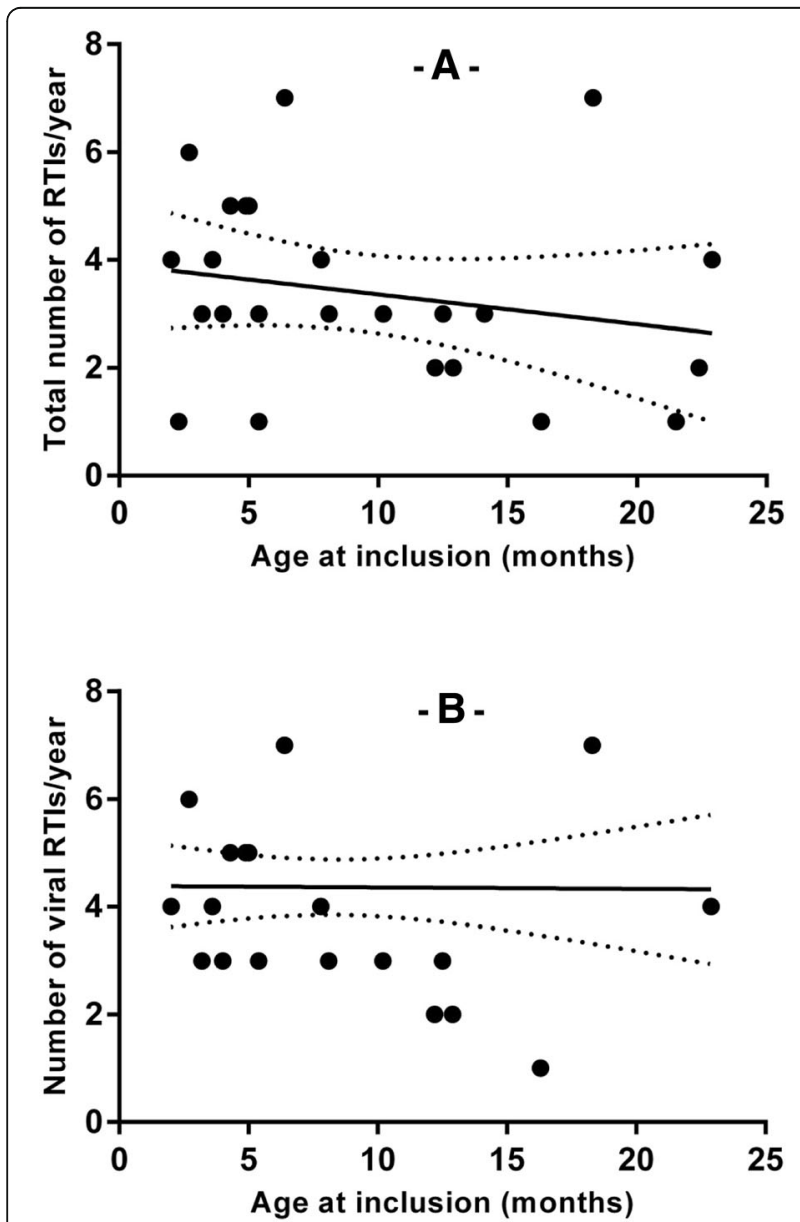

Fig. 1 a Correlation between age at inclusion and total respiratory tract infection (RTI) episodes; b Correlation between age at inclusion and virus-positive respiratory tract infection (RTI) episodes co-infections were identified during RTIs; one of them required hospitalization (Table 2).

For the six patients presenting a RSV infection, the clinical progression was uncomplicated (Table 3).

\section{Viruses recovered without RTI}

One-third of the viral samples collected outside RTI episodes were virus-positive (Table 2). Compared to those recovered during RTI, the proportions were quite similar for RV, adenoviruses and metapneumovirus. However, a smaller proportion of RSV and influenza but a higher proportion of bocavirus and coronavirus were found in nasal swabs collected outside RTI periods.

\section{Comparison of virus-positive and -negative RTI outcomes (Table 4)}

No differences were found between virus-positive and -negative RTIs among the following outcome measures: age at inclusion, weight Z-score at inclusion, difference of weight Z-scores between the beginning and end of the study period, total number of RTIs and cumulative number of days of antibiotics.

\section{Viral RTI-related healthcare consumption}

One hospitalization was directly related to a viral RTI (LRTI related to RV/EV-adenovirus co-infection) out of the five hospitalizations. The others causes of hospitalization were non-respiratory viral infection (gastroenteritis) in two cases, one for prolonged cardiorespiratory monitoring and one for surgery (hexadactylia).

Supplemental medications were needed in the vast majority of viral RTIs: oral antibiotics were prescribed at least once in 22 patients (88\%) for a mean length of $13.8 \pm 6.2$ days and a mean cumulative number of days of $28.9 \pm 21$ days. Oral steroids were used in five patients (21\%) for a mean length of $4.4 \pm 1.4$ days because of prolonged or recurrent wheezing spells, and bronchodilators were used in four $(18 \%)$ for a mean length of $4.5 \pm 7.8$ days. A significant correlation was found between the total number of RTIs (virally proven or not) and the cumulative number of days under antibiotics used during the year of follow-up (Pearson correlation, $r=0.7941$, $p<0.0001)$.

Viral RTI was associated with a mean $0 \pm 0.2$ days of parental absenteeism.

\section{Nutritional impact}

At the beginning of the study the mean weight Z-score was $-1.3 \pm 1.4$; it was $-0.9 \pm 1.6$ at the end of the study. There was no correlation between weight Z-score differences between the start and the end of the study and the number of viral RTIs occurring during the year (Pearson correlation, $r=-0.1708, p=0.4142$ ). 


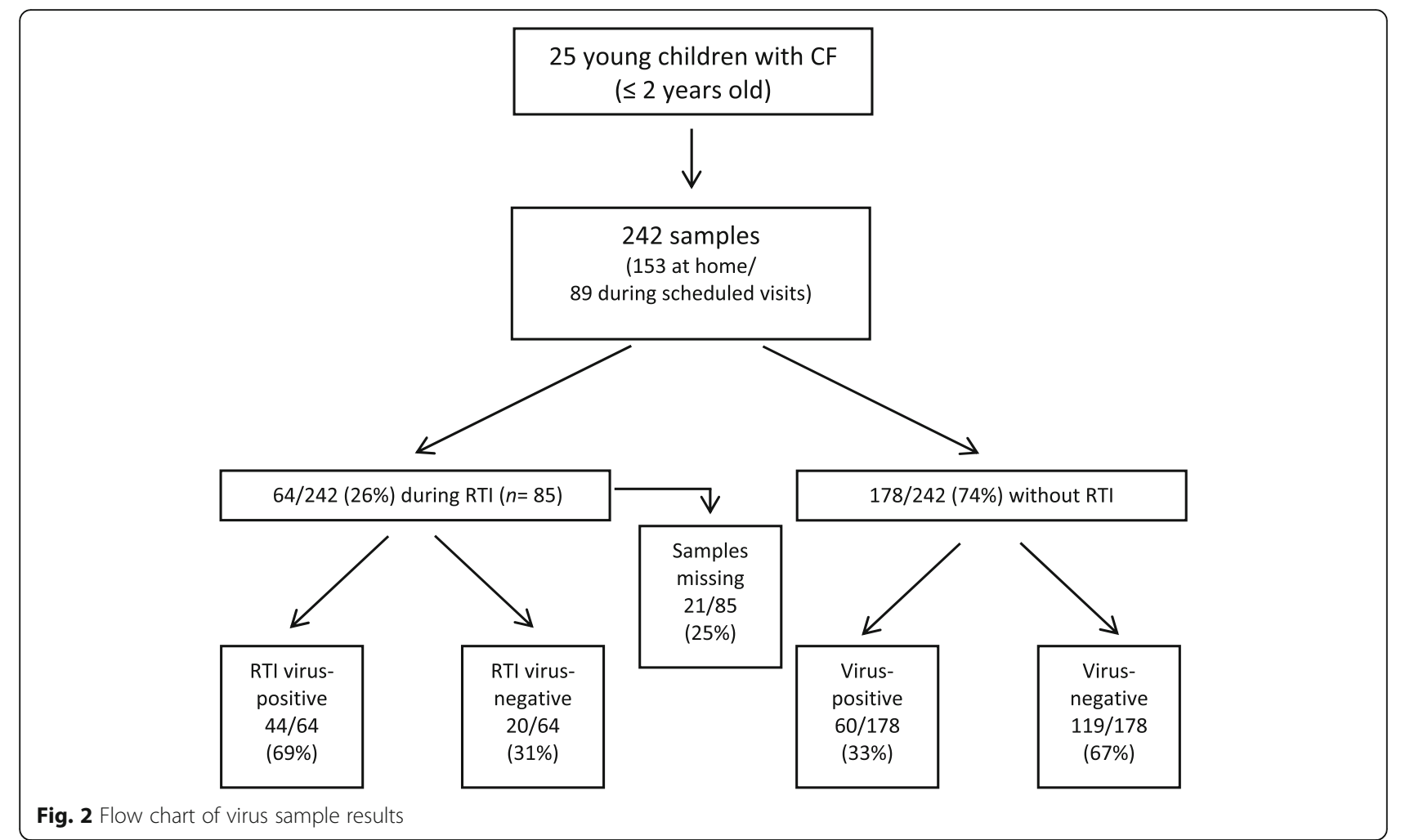

\section{New pathogen acquisition}

Four percent of the patients were positive for PA and $32 \%$ for SA at inclusion. A mean $5.6 \pm 1.5$ swabs were taken during the scheduled visit at the center for bacterial analysis. During the study, new isolation of PA occurred in five patients $(5 / 24 ; 21 \%)$ and in ten patients $(10 / 17 ; 59 \%)$ for SA. It is noteworthy that while none of our participants had Stenotrophomonas maltophilia (SM) at inclusion, four acquired this bacterium during the study period. The small number of patients in each

Table 2 Viruses recovered during RTI and without RTI

\begin{tabular}{lll}
\hline & During RTI $(n=44)$ & Without RTI $(n=60)$ \\
\hline RV/EV & $27(61 \%)$ & $31(52 \%)$ \\
Adenovirus & $6(14 \%)$ & $9(15 \%)$ \\
RSV A/B & $6(14 \%)$ & $2(3 \%)$ \\
Influenza A/B & $4(9 \%)$ & $1(2 \%)$ \\
Bocavirus & $3(7 \%)$ & $13(22 \%)$ \\
Parainfluenza 1-4 & $2(5 \%)$ & - \\
Metapneumovirus & $1(2 \%)$ & $1(2 \%)$ \\
Coronavirus $^{a}$ & - & $9(15 \%)$ \\
Co-infections $^{\text {a }}$ & $8(20 \%)$ & $8(13 \%)$
\end{tabular}

${ }^{a}$ co-infections during RTI: rhinovirus associated with adenovirus $(n=2)$, RSV $(n=1)$, bocavirus $(n=1)$; bocavirus associated with influenza $(n=1)$, adenovirus $(n=1)$ and adenovirus associated with RSV $(n=3)$ Co-infections without RTI: rhinovirus associated with adenovirus $(n=2)$, bocavirus $(n=2)$, coronavirus $(n=1)$; bocavirus associated with adenovirus $(n=1)$, influenza $(n=1)$, cytomegalovirus $(n=1)$ subgroup precluded further statistical analysis. However, there was a trend for a higher number of total RTIs in patients with new isolations of PA, and the same was noticed for SM (Table 5).

\section{Discussion}

\section{RTI epidemiology in young children with CF}

Until recently, there were very few data describing RTI frequency, clinical consequences and nature of the virus recovered in young patients ( $\leq 2$ years) with $\mathrm{CF}$ using molecular diagnostic tools [6]. A consortium has been set up between the USA and Australia to precisely evaluate the impact of early respiratory viral infections in young children with CF (NCT01973192). We found that a mean 3.4 RTI episodes occurred in our patients over 1 year; the maximum number of RTIs recorded was seven in one patient. This frequency is in accordance with data reported by Collinson et al. in the late 1990s (3.4 URTIs per year in children under 6 years of age) using cultural or serological tools [18], but is half the 3.8 episodes identified using PCR over the 6 winter months reported by van Ewijk et al. in a population of children with $\mathrm{CF}$ who were a mean 3.5 years old [9]. The most recent data published by the Swiss group did not provide a precise RTI frequency calculation because their methodology differed from ours [6]. The reason for the LRTI frequency reported in our study likely stems from the scoring system itself and the way we used it for the purposes 
Table 3 RSV infected patient's characteristics

\begin{tabular}{|c|c|c|c|c|c|c|}
\hline \multirow[t]{2}{*}{ Patient number } & \multirow[t]{2}{*}{ Age at inclusion (months) } & \multirow[t]{2}{*}{ Age at RSV infection (months) } & \multicolumn{4}{|l|}{ Requirements for extra-care } \\
\hline & & & Hospitali-zation/ Oxygen supply & Antibiotics & $\mathrm{SABD}^{\mathrm{C}}$ & Oral steroids \\
\hline 1 & 16.3 & 22.9 & No/No & Yes & Yes & No \\
\hline 2 & 8.1 & 19.6 & No/No & Yes & Yes & Yes \\
\hline 3 & 4.0 & 12.8 & No/No & Yes & Yes & Yes \\
\hline 4 & 2.0 & $14.1^{\mathrm{a}}$ & No/No & Yes & No & No \\
\hline 5 & 2.7 & $4.5^{\mathrm{b}}$ & $\mathrm{No} / \mathrm{No}$ & Yes & No & No \\
\hline 6 & 3.2 & $12.3^{\mathrm{a}}$ & No/No & Yes & No & Yes \\
\hline
\end{tabular}

${ }^{a}$ co-infected with RVh

${ }^{\mathrm{b}}$ co-infected with adenovirus

'SABD short acting bronchodilators

of the study. This so-called "respiratory illness" scoring system was developed by van Ewijk et al. for parents for self-recording of URTI and/or LRTI clinical symptoms. This clinical score was systematically measured twice weekly. In the present study, because of the high risk of errors in filling in the diary or giving up over a longer period of time (12 months vs. 6 in the van Ewijk et al. study), we chose not to use it on a systematic basis, but rather as an aid for both parents and home caregivers to detect RTIs and launch nasal sampling. Furthermore, it appears that some RTI episodes may have been missed using this score, particularly mild URTIs.

As expected in this young population, RV/EV remain the most frequently recovered viruses during RTI. Since the spread of molecular diagnostic tools, many studies have reported the same results in older children $[1,4,6,9,15,25,26]$, adolescents and adults $[3,10,11,15]$. Overall, RV/EV are responsible for 70 $80 \%$ of virally induced RTIs. However, we were more intrigued by the few RSV infections detected. Six patients were infected by RSV at a mean age of $14 \pm 6.4$ months; none required hospitalization, and even in the youngest patients, the clinical course was uncomplicated. These findings contrast with those reported decades ago in unscreened CF babies in whom RSV infection was described as severe (requiring oxygen supply or invasive ventilation) in three out of seven patients $(43 \%)[16,17,27]$. The overall better health status of the children with $\mathrm{CF}$ in the present sample is likely the main explanation for this result, as well as their older age at the time of RSV infection. These results are particularly interesting in terms of RSV infection prevention and subsequent clinical trials. Our population of patients was palivizumab-free. The American Academy of Pediatrics recommends the use of palivizumab in CF for targeted patients, particularly those with poor nutritional status or with several siblings [28]. We speculate that precautions and preventive measures (hand washing, family daycare attendance, avoidance of virally infected individuals, etc.) followed by parents (on medical advice and on their own initiative) may be efficient enough to reduce virus circulation around these patients.

\section{RV/EV carriage}

Approximately one-third of the study population carried viruses without any symptoms. RV/EV were recovered in $51 \%$ of these cases. The clinical significance of this finding is currently unknown, as are the underlying mechanisms and potential consequences. Whether or not this was the same subtype of picornavirus (RV or EV) was not addressed in this study, but prolonged carriage has been reported $[4,29]$. In a recent study conducted in a mean of

Table 4 Comparison of outcomes in virus-positive and -negative RTIs

\begin{tabular}{|c|c|c|c|}
\hline Outcome measures & Virus (-) RTI & Virus (+) RTI & $P$-value \\
\hline Number of episodes & $(n=20)$ & $(n=44)$ & \\
\hline Age at inclusion (months) & $7.1 \pm 1.4$ & $8.3 \pm 0.9$ & 0.4668 \\
\hline Total number of RTI episodes & $4.6 \pm 0.3$ & $4.3 \pm 0.2$ & 0.6037 \\
\hline Respiratory symptom duration (days) & $7.8 \pm 0.5$ & $8.3 \pm 0.5$ & 0.9585 \\
\hline Weight Z-score at inclusion & $-1.4 \pm 0.3$ & $-1.4 \pm 0.2$ & 0.1166 \\
\hline Difference of weight Z-scores ${ }^{a}$ & $-0.5 \pm 0.2$ & $0.3 \pm 0.1$ & 0.5703 \\
\hline Cumulative number of days of antibiotics $^{\mathrm{b}}$ & $41.1 \pm 5$ & $37.8 \pm 3.2$ & 0.5646 \\
\hline
\end{tabular}

Results are presented as mean \pm SD. Wilcoxon signed rank for paired comparisons

a'Weight Z-score at the end of the study period - weight Z-score at the beginning

${ }^{b}$ Over the year of the study follow-up 
Table 5 Microbiological outcome measures

\begin{tabular}{lllllll}
\hline & $\mathrm{PA}+$ & $\mathrm{PA}-$ & $\mathrm{SA}+$ & $\mathrm{SA}-$ & $\mathrm{SM}+$ & $\mathrm{SM}-$ \\
\hline$n$ & 5 & 20 & 10 & 15 & 4 & 21 \\
Total RTI number & $4.2 \pm 1.8$ & $3.2 \pm 1.7$ & $3.3 \pm 2.1$ & $3.5 \pm 1.6$ & $4.8 \pm 1.7$ & $3.1 \pm 1.7$ \\
Total virus positive RTI & $1.0 \pm 1.22$ & $2.0 \pm 1.6$ & $1.1 \pm 2.3$ & $2.3 \pm 1.5$ & $2.5 \pm 2.4$ & $1.7 \pm 1.4$ \\
\hline
\end{tabular}

$\mathrm{PA}+$ : patient with new isolation of Pseudomonas aeruginosa (PA) during the study period

PA-: patient without new isolation of Pseudomonas aeruginosa (PA) during the study period

$\mathrm{SA}+$ : patient with new isolation of Staphylococcus aureus (SA) during the study period

SA-: patient without new isolation of Staphylococcus aureus (SA) during the study period

$\mathrm{SM}+$ : patient with new isolation of Stenotrophomonas maltophilia (SM) during the study period

SM-: patient without new isolation of Stenotrophomonas maltophilia (SM) during the study period

3.5 years old (range, $0-17$ years) CF patients population sampled weekly for 6 months, the authors found that patients with CF had a more frequent and prolonged carriage of RV in comparison to their healthy counterparts, even if the subtypes did not differ [4]. The authors conclude that "this may indicate increased viral replication and/or decreased viral antiviral defense in patients with CF." In younger patients (included within their first 3 months of life), Korten et al. found that RV without symptoms was detected in $20 \%$ of cases and that virus detection was less frequently associated with symptoms in comparison to healthy infants. This raises the question of the pathogenicity of these viruses in young patients with CF.

\section{Viral-bacterial interactions}

We found that there was a trend toward a higher frequency of total RTI in young children in whom the first isolation of PA and SM occurred during the year of follow-up. However, we did not find the same trend for SA. However the study was not powered to specifically address this question, thus preventing us from drawing a firm conclusion. The risk factor that RTI represents for PA colonization was underlined several years ago, particularly after a severe episode of RTI $[17,27]$. Furthermore, major pathogen acquisition in young children with CF (PA, SA, Achromobacter xylosoxidans) may follow seasonality, underpinning the concept of predisposing seasonal environmental factors such as respiratory virus epidemics [30, 31]. However, the trend identified herein for SM seems somewhat new and will need further attention. Whether viruses are able to directly alter airway bacterial flora in young patients with CF or these alterations are related to the frequent usage of antibiotics remains to be determined.

\section{Strengths and limitations of the study}

This study's strengths include its prospective cohort design, one of the first such studies conducted in young children with palivizumab-free $\mathrm{CF}$, preventing potential interference of the monoclonal antibody on respiratory viral epidemiology. At home, samples were taken by physiotherapists using a detailed protocol, ensuring collection of good-quality samples.
This study also has limitations. (1) There were fewer samples in comparison to recent studies $[4,6]$. (2) In our hands, despite the presence of healthcare providers at home, the scoring system used to help parents and professional caregivers identify RITs in children was weak and may have missed several mild RTIs. (3) The overall low number of samples in each subgroup make the analysis of some well-known risk factors of RTIs such as daycare attendance or tobacco smoke exposure impossible. (4) The subjects' median age at inclusion was 6.4 months, which is likely already "too late" to evaluate the clinical impact of some viral RTIs that are known to be more worrisome at the youngest ages (usually before 3 months of age). (5) Finally, healthy controls were not included for comparison purposes.

\section{Conclusion and future work}

This study shows that respiratory viruses are responsible for around two-thirds of RTIs in young children with CF. In contrast to older studies and in accordance with more recent studies, viral RTIs are usually of mild severity, exceptionally leading to hospitalization. RSV infections were rare, and this raises the question of the usefulness of RSV preventive medication in this young population.

\section{Abbreviations \\ CF: Cystic fibrosis; EV: Enterovirus; LRTI: Lower RTI; PA: Pseudomonas aeruginosa; RSV: Respiratory syncytial virus; RTI: Respiratory tract infection; RV: Rhinovirus; SA: Staphylococcus aureus; URTI: Upper RTI}

\section{Acknowledgements}

Not applicable

\section{Authors' contributions}

ME: data collection and analysis, manuscript writing and review. FM: data analysis, results discussion, manuscript review. AD-J: data analysis, results discussion, manuscript review. MP: database work-up and data collection. CO: results discussion, manuscript review. CM: results discussion, manuscript review. PR: study design, data analysis, manuscript writing and review. All authors read and approved the final manuscript.

\section{Funding}

This study was funded by two grants from the Association Logistique Lyonnaise Post Hospitalière in 2013 and 2014. These fundings were used to support the cost of virologic analyses. 


\section{Availability of data and materials}

The datasets used and/or analysed during the current study are available from the corresponding author on reasonable request.

\section{Ethics approval and consent to participate}

All procedures performed in studies involving human participants were in accordance with the ethical standards of the institutional and/or national research committee and with the 1964 Helsinki Declaration and its later amendments or comparable ethical standards. The study was approved by the local Institutional Review Board (Comité Consultatif de Protection des Personnes dans la Recherche Biomédicale, Lyon; No. 2014-AO1387640). Informed consent to participate was obtained from all individual participants (or their legal representative) included in the study.

\section{Consent for publication}

Informed consent for publication was obtained from all individual participants (or their legal representative) included in the study.

\section{Competing interests}

The authors declare that they have no competing interests.

\section{Author details}

${ }^{1}$ Service de pneumologie pédiatrique et CRCM enfant, Hôpital Femme Mère Enfant, Hospices civils de Lyon, Bron, France. 'Laboratory of Virology, Institut des Agents Infectieux, Groupement Hospitalier Nord, F69317 Lyon, France. ${ }^{3}$ Centre National de Référence des virus respiratoires France Sud, Hospices Civils de Lyon, Lyon, France. ${ }^{4}$ Faculté de Pharmacie, CIRI, Inserm U1111 CNRS UMR5308, Virpath, Univ Lyon, Université Lyon 1, Lyon, France. ${ }^{5}$ Equipe de Recherche, Bactéries Pathogènes Opportunistes et Environnement, UMR CNRS 5557 Ecologie Microbienne, Université Lyon 1 \& VetAgro Sup, Villeurbanne, France. 'Laboratory of Bacteriology, Institut des Agents Infectieux, Groupement Hospitalier Nord, Hospices Civils de Lyon, F69317 Lyon, France. ${ }^{7}$ UMR 5558 (EMET). CNRS, LBBE, Université de Lyon, Villeurbanne, France. ${ }^{8}$ Centre de ressources et de compétence pour la Mucoviscidose, 59 boulevard Pinel, 69677 BRON CEDEX, France.

Received: 20 September 2018 Accepted: 25 July 2019

\section{Published online: 03 September 2019}

\section{References}

1. Asner $S$, Waters $V$, Solomon $M$, et al. Role of respiratory viruses in pulmonary exacerbations in children with cystic fibrosis. J Cyst Fibros. 2012;11(5):433-9.

2. Burns JL, Emerson J, Kuypers J, et al. Respiratory viruses in children with cystic fibrosis: viral detection and clinical findings. Influenza Other Respir Viruses. 2012;6(3):218-23.

3. Cousin M, Molinari N, Foulongne V, et al. Rhinovirus-associated pulmonary exacerbations show a lack of FEV1 improvement in children with cystic fibrosis. Influenza Other Respir Viruses. 2016;10(2):109-12.

4. Dijkema JS, van Ewijk BE, Wilbrink B, et al. Frequency and duration of rhinovirus infections in children with cystic fibrosis and healthy controls: a longitudinal cohort study. Pediatr Infect Dis J. 2016;35(4):379-83.

5. Kieninger $E$, Singer $F$, Tapparel $C$, et al. High rhinovirus burden in lower airways of children with cystic fibrosis. Chest. 2013;143(3):782-90.

6. Korten I, Kieninger E, Klenja S, et al. Respiratory viruses in healthy infants and infants with cystic fibrosis: a prospective cohort study. Thorax 2017.

7. Miro-Canis S, Capilla-Rubio S, Marzo-Checa L, et al. Multiplex PCR reveals that viruses are more frequent than bacteria in children with cystic fibrosis. J Clin Virol. 2017;86:1-4.

8. Muller L, Mack I, Tapparel C, et al. Human rhinovirus types and association with respiratory symptoms during the first year of life. Pediatr Infect Dis J. 2015;34(8):907-9.

9. van Ewijk BE, van der Zalm MM, Wolfs TF, et al. Prevalence and impact of respiratory viral infections in young children with cystic fibrosis: prospective cohort study. Pediatrics. 2008;122(6):1171-6.

10. Etherington $C$, Naseer $R$, Conway SP, et al. The role of respiratory viruses in adult patients with cystic fibrosis receiving intravenous antibiotics for a pulmonary exacerbation. J Cyst Fibros. 2014;13(1):49-55.

11. Flight WG, Bright-Thomas RJ, Tilston $P$, et al. Incidence and clinical impact of respiratory viruses in adults with cystic fibrosis. Thorax. 2014;69(3):247-53.

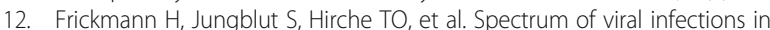
patients with cystic fibrosis. Eur J Microbiol Immunol (Bp). 2012;2(3):161-75.
13. Goffard A, Lambert V, Salleron J, et al. Virus and cystic fibrosis: rhinoviruses are associated with exacerbations in adult patients. J Clin Virol. 2014;60(2): 147-53.

14. Hoek RA, Paats MS, Pas SD, et al. Incidence of viral respiratory pathogens causing exacerbations in adult cystic fibrosis patients. Scand J Infect Dis. 2013:45(1):65-9.

15. Wark PA, Tooze $M$, Cheese $L$, Whitehead $B$, et al. Viral infections trigger exacerbations of cystic fibrosis in adults and children. Eur Respir J. 2012; 40(2):510-2

16. Abman SH, Ogle JW, Butler-Simon N, et al. Role of respiratory syncytial virus in early hospitalizations for respiratory distress of young infants with cystic fibrosis. J Pediatr. 1988;113(5):826-30.

17. Armstrong D, Grimwood K, Carlin JB, et al. Severe viral respiratory infections in infants with cystic fibrosis. Pediatr Pulmonol. 1998;26(6):371-9.

18. Collinson J, Nicholson KG, Cancio E, et al. Effects of upper respiratory tract infections in patients with cystic fibrosis. Thorax. 1996;51(11):1115-22.

19. Ramsey BW, Gore EJ, Smith AL, et al. The effect of respiratory viral infections on patients with cystic fibrosis. Am J Dis Child. 1989;143(6):662-8.

20. Somayaji R, Goss CH, Khan U, et al. Cystic fibrosis pulmonary exacerbations attributable to respiratory syncytial virus and influenza: a population-based study. Clin Infect Dis. 2017;64(12):1760-7.

21. Metz J, Eber E, Resch B. Respiratory syncytial virus infection-associated hospitalization rates in infants and children with cystic fibrosis. Pediatr Infect Dis J. 2017:36(6):545-8.

22. Buchs $C$, Dalphin ML, Sanchez $S$, et al. Palivizumab prophylaxis in infants with cystic fibrosis does not delay first isolation of Pseudomonas aeruginosa or Staphylococcus aureus. Eur J Pediatr. 2017;176(7):891-7.

23. Gangell CL, Shackleton C, Poreddy S, et al. Feasibility of parental collected nasal swabs for virus detection in young children with cystic fibrosis. J Cyst Fibros. 2014;13(6):661-6.

24. Emerson J, Cochrane E, McNamara S, et al. Home self-collection of nasal swabs for diagnosis of acute respiratory virus infections in children with cystic fibrosis. J Pediatric Infect Dis Soc. 2013;2(4):345-51.

25. Kieninger $\mathrm{E}$, Vareille $\mathrm{M}$, Kopf BS, et al. Lack of an exaggerated inflammatory response on virus infection in cystic fibrosis. Eur Respir J. 2012;39(2):297-304.

26. Esther CR Jr, Lin FC, Kerr A, et al. Respiratory viruses are associated with common respiratory pathogens in cystic fibrosis. Pediatr Pulmonol. 2014;49(9):926-31.

27. Hiatt PW, Grace SC, Kozinetz CA, et al. Effects of viral lower respiratory tract infection on lung function in infants with cystic fibrosis. Pediatrics. 1999;103(3):619-26.

28. American Academy of Pediatrics Committee on Infectious D, American Academy of Pediatrics Bronchiolitis Guidelines C. Updated guidance for palivizumab prophylaxis among infants and young children at increased risk of hospitalization for respiratory syncytial virus infection. Pediatrics. 2014; 134(2):e620-38

29. Flight WG, Bright-Thomas RJ, Tilston $P$, et al. Chronic rhinovirus infection in an adult with cystic fibrosis. J Clin Microbiol. 2013;51(11):3893-6.

30. Psoter KJ, De Roos AJ, Wakefield J, et al. Season is associated with Pseudomonas aeruginosa acquisition in young children with cystic fibrosis. Clin Microbiol Infect. 2013;19(11):E483-9.

31. Psoter KJ, De Roos AJ, Wakefield J, et al. Seasonality of acquisition of respiratory bacterial pathogens in young children with cystic fibrosis. BMC Infect Dis. 2017;17(1):411.

\section{Publisher's Note}

Springer Nature remains neutral with regard to jurisdictional claims in published maps and institutional affiliations. 